Article

\title{
Leading Indicators-A Conceptual IoT-Based Framework to Produce Active Leading Indicators for Construction Safety
}

\author{
Aaron Costin*(D), Andrew Wehle and Alireza Adibfar \\ M. E. Rinker, Sr. School of Construction Management, University of Florida, 344 Rinker Hall, Gainesville, \\ FL 32611, USA; wehleaj@ufl.edu (A.W.); adib2016@ufl.edu (A.A.) \\ * Correspondence: aaron.costin@ufl.edu
}

Received: 8 September 2019; Accepted: 11 December 2019; Published: 16 December 2019

\begin{abstract}
Active leading indicators (ALIs) have the potential to identify safety hazards and prompt immediate actions to prevent incidents. Currently, there is a major gap in research that incorporates a fully automated ALI system because implementation has been hindered by a lack of established industry thresholds of measurable performance that would trigger an actionable response. Therefore, this paper addresses this gap by presenting a new method that utilizes the Internet of Things (IoT) to collect quantifiable data which can trigger an actionable response in real time based on established thresholds. This novel method integrates the Construction Industry Institute (CII) active leading indicator framework with a prototype IoT-based system. Significantly, the ALI provides the physical-virtual feedback loop, which is an essential aspect of the IoT system because it provides real-time feedback to both the users and systems. This paper also identifies potential inputs to the ALI framework from emerging IoT-enabled systems. A case study was presented to initially validate the IoT-based ALI framework. Bluetooth-enabled heart rate monitors were issued to workers in a hazardous and critical mining construction site. The ALIs that were recorded included heart rate and body temperature. Thresholds were established that alerted the monitoring safety staff when a worker exhibited potentially unsafe conditions. The results of the study demonstrated the feasibility of the system. Additionally, other results included worker resistance; non-disclosing of medical conditions, and limitations for IoT connectivity.
\end{abstract}

Keywords: active leading indicators; leading indicators; construction safety; Internet of Things; safety management; Incident of Concern (IOC)

\section{Introduction}

Maintaining a high level of safety is the utmost importance for the well-being of construction workers and citizens in close proximity to an active jobsite. Injury and death are common occurrences in construction. Construction is one of the largest and most important industries in the U.S.; it is also one of the most dangerous industries to work in. In the past two decades, more than 28,000 U.S. construction workers have died at work. According to the U.S. Department of Labor, 971 construction workers were killed in the U.S. in 2017, accounting for $20.7 \%$ of workplace fatalities for all industries [1]. With every single fatality, there are hundreds more being seriously injured. Approximately $90 \%$ of workplace injuries can be traced to unsafe work practices and behaviors. As these statistics indicate, safety hazards in the construction process remain a major problem for the entire community beyond the workforce. The sad reality of frequent loss of life, injuries, near-misses, and collateral damages poses liabilities that can be prevented. Significantly worse, construction can pose safety risks to the surrounding inhabitants going about their daily lives; and this often occurs without warning [2]. 
Even with high-performing safety projects, construction still poses many hazards to the surrounding inhabitants, including delays, unsightly scenes, pollution, noise, and environmental hazards which can be detrimental to the economy and to the quality of life of the greater community.

The U.S. construction industry is one of the largest markets in the nation and makes up a substantial portion of the U.S. economy. In 2016, the construction industry contributed to $6.1 \%$ of all industry employment and $4.2 \%$ of the gross domestic product (GDP) [3]. A major motivation for increasing safety is that safety has a direct impact on production as well as the profitability of a project. The costs of safety incidents can be detrimental to a company's financial viability. Incidents have high direct costs (medical bills, increased insurance premiums, workers compensation, legal claims, fines, etc.), and indirect costs can also be staggering. Indirect costs include the loss of time due to the project being shut down, increasing safety training and education programs, a decrease in worker morale, and potential lost revenue from not being selected on future projects due to safety incident rates. The Occupational Safety and Health Administration [4] provides a tool and program to calculate the direct and indirect costs of workplace injuries and illnesses. The tool utilizes the National Council on Compensation Insurance, Inc. (NCCI) average claim data, a company's profit margin, the average costs of an injury or illness, and an indirect cost multiplier to project the amount of sales a company would need to generate to cover those costs. For example, a simple sprain will have a direct cost of $\$ 29,989$ and an additional indirect cost of $\$ 32,987$, which would require the company to make an addition sale of $\$ 2,099,200$ (at $3 \%$ profit) to recuperate the costs. "Zero incident" goals are a common goal among construction companies, since each incident is very costly in terms of time, money, and human suffering.

Traditionally, safety performance in the construction industry has been measured by metrics such as the Occupational Safety and Health Administration (OSHA) recordable injury rate (RIR); days away, restricted work, or transfer (DART) injury rate; or the experience modification rating (EMR) associated with workers' compensation insurance. These metrics have served the purpose of providing information by which contractors could assess their safety performance in terms of construction industry averages on those metrics or could make comparisons with other firms. These metrics have also been used widely by OSHA, insurance companies, facility owners, and other parties involved in construction projects. Other metrics that are used by the industry include loss ratios (ratio of the cost of claims to the cost of premiums), the number of liability claims associated with worksite injuries, and the number of OSHA citations/fines. However, these traditional metrics provide historical information about some aspect of the measures of safety performance that has occurred. These particular measures are defined and classified as lagging indicators [5]. Traditional measures are considered lagging because they are derived and linked to an incident or event that occurred in the past. Traditional safety metrics are out of date given the current abilities to collect, analyze, and share safety data. The entire workforce could benefit from the integration of new technologies to collect real-time information and improved indicator data, rather than rely on post-accident analysis.

Lagging indicators may not be the most effective means to prevent future occurrences of accidents and injuries. Given the rapid development of new technologies, there are now better means and methods to measure and assess safety and worker behaviors over the entire course of a given project. In contrast, leading indicators (LI) are measures that can be used as predictors of future levels of safety performance because they reflect information about the safety process prior to the occurrence of a safety incident $[5,6]$. A critical distinction regarding leading indicators is the ability to use the information gathered to affect change prior to an injury or accident. The development and implementation of new technologies with the ability to provide timely data assist safety managers to help determine breakdowns in safety performance and as a result respond to emerging data as a means to address safety issues prior to the occurrence of a serious event or incident. When one or more of these measures suggest that some aspect of the safety process is weak or weakening, interventions can be implemented to improve the safety process and, thereby positively impact the safety process before any negative occurrences (injuries) are sustained [5]. 
Leading indicators are designed to monitor construction safety processes and worker behaviors. When the measures show that there is a flaw in the process some type of intervention is initiated. Leading indicators provide a means of tracking or monitoring the performance of a process as it is taking place, or they provide a way of showing whether a particular process or processes are being implemented as planned. If flaws are noted in the process of implementation, there is an increased possibility of injury occurrence. An intervention is then initiated to make corrections in the process prior to an injury or catastrophic event.

The authors of [5] define LIs in a complex way based on the type of data collected and the expected predictive value of the measure creating and defining the terms passive and active leading indicators. Passive leading indicators (PLIs) are measurements or information streams that provide an indication of the probable safety performance to be realized within a firm or on a project. While PLIs have been reported to be predictive on a macro scale, they have limited predictive value after a certain point in time or once a threshold is reached. They are considered passive in nature because they are often measured as a precursor to people entering the work environment and updates are not actively sought out after they have been initially measured.

Active leading indicators (ALIs) are measurements or information streams that also provide an indication of the probable safety performance to be realized within a firm or on a project; however, they are dynamic and thus more subject to change in a short period of time [5]. ALIs may be characterized as the "pulse" of the construction project in terms of daily safety behaviors and practices. In order to have predictive value, ALIs must be actively sought in terms of timely and accurate reports, measurements, and observations.

Although ALIs are better suited for preventing accidents, [5] reports that ALI implementation has been hindered by a lack of established industry thresholds of measurable performance that would trigger an actionable response. The Construction Industry Institute (CII) report discusses the implementation of active leading indicators [7]. However, the technologies that can produce real-time active indicators that are quantifiable and measurable did not exist at the time of the $2012 \mathrm{CII}$ study. Ultimately, the active LI framework has not been fully implemented to date. Therefore, the purpose of this research is to develop a framework that can produce, collect, and analyze real-time quantifiable ALIs. The utilization of emerging Internet of Things (IoT) technology is proposed to resolve this challenge through the collection of quantifiable data that can trigger an actionable response in real time based on established thresholds.

\section{Literature Review}

\subsection{Safety Culture}

Safety professionals need to provide management with the knowledge and research on leading indicators in a way that generates greater awareness and demonstrative support for safety participation throughout the organization. In order for the initiatives and activities of a comprehensive safety program to be successful, the program must be effectively created, communicated, taught, and reported throughout the chain from the owner through management to the worker on the site. Due to the dynamic and complex nature of modern safety programs, it is common for safety professionals to refer to this as a safety culture. The term safety culture was first published in the 1987 Organization for Economic Co-operation and Development (OECD) Nuclear Agency report regarding the 1986 Chernobyl power plant incident [8]. In this particular case, the accident report suggests that a prevailing negative attitude in regard to management and organization contributed to the root causes of the accident, fostering a safety culture of complacent neglect associated with technical and individual human errors. Since 1986, the concept of safety culture has been researched and applied to multiple industries.

There are several other definitions of safety culture. Safety culture has also been defined set of norms, beliefs, values and attitudes that are learned and shared by individual as well as groups of people [9]. The International Atomic Energy Authority (IAEA) defines it as an assembly of 
characteristics and attitudes in organizations that establishes safety issues as a central priority [8]. The environment of any construction site is composed of individuals who may take risks, obey rules as well as break rules and procedures. Safety culture is an adaptive tool that when properly designed, communicated and implemented, seeks to mitigate the risk factors and establish a set of obligations and expectations that prevail throughout the project.

The author of [10] reports on the importance of safety culture, especially in regard to its impact on human behavior in the field. The author notes, in the study of the mining industry, that very few accidents occurred that was not addressed by a previously established rule or procedure in place. In order to improve safety, management need to utilize methods of safety management that go beyond simply developing rules and procedures. If management want to more effectively impact employee behavior in regard to safety, safety staff will generally have to go down the chain of command to the grass roots level in order to more accurately determine how/why things are actually done at the worker level. A positive safety culture provides a platform from which to better administer the safety program as well as gain access to indicators regarding awareness, understanding, cooperation and compliance with rules and regulations.

Related to safety culture, [11] conduct an examination of safety climate. Based on the work of [12-14], the research suggests that individuals who perceive that their organization values safety are more likely to follow established safety procedures. Employees who engage in work environments featuring a strong safety climate tend to voluntarily engage in safety activities and are less likely to be involved in safety incidents [11]. Empowerment and employee involvement in safety decision making encourages workers to take responsibility for their behavior resulting in positive safety behavior outcomes [14,15].

\subsection{Leading Indicator Data}

Leading indicators (LIs) consist of a set of selected measures that describe the level of effectiveness of the safety process and are linked to actions taken to prevent accidents [16]. The authors of [17] describe LIs as conditions, events, or measures that precede an incident and that have a predictive value in regard to unsafe working conditions or behaviors. When one or more of these measures suggest that some aspect of the safety process is weak or weakening, interventions can be implemented to improve the safety process and thereby positively impact the safety process before any negative occurrences (injuries) are sustained [5]. According to [18], leading indicators should be used by safety professionals to communicate safety goals throughout the entire organizational hierarchy, particularly addressing the roles, goals, and obligations of senior management. When leading indicators become a priority at the highest level, the elevated importance of site safety permeates throughout the organization, fostering the use and acceptance of leading indicators as a tool to support safety.

The authors of [17] acknowledge the interrelationship between leading indicators and safety culture. For leading indicators to be recognized as precursors to incidents, there has to be an established, learned, and shared understanding of what is normal, expected, and desired versus what is not normal, unexpected, or undesirable [17]. The movement to higher levels of safety requires that people who work on the site have collectively learned and as a result have a shared understanding of what to look out for, monitor, expect and fear when experiencing indicators in the field. It is the identification of leading indicators and their utilization within a shared and integrated safety culture that helps communicate and implement in action the shared model of safety throughout an organization. Critical to the progressive definition of these measures are the creation and monitoring of indicators that allow for early detection of failures in the safety management and safety culture [19]. Leading indicators function as early detection measures and clearly fulfill a critical role within the safety culture of an organization. According to the International Nuclear Safety Advisory Group (INSAG), safety management is comprised of the arrangements made by the organization for the management of safety of the entire project in order to promote a strong safety culture resulting in good safety performance [19]. Central to this definition by the INSAG is the established connection between "safety 
management" as an organization and "safety culture" in which the entire operation operates. In their conceptualization, safety management and safety culture are inseparable. In order to effectively manage safety, management must utilize an integrated and systematic approach that is deliberately aware of the effects of their actions on individual as well as the collective human behavior of people within the system [19].

The authors of [19] report that high levels of safety in the nuclear industry are a result of the complex interactions between engineered safety and operational safety. A focus on any single aspect of safety performance is ineffective and misleading. The utilization of both leading and lagging indicators provides a more valid picture of overall operational safety performance. LIs are most effective when they are used as a precursor measurement of site safety degradation, prompting action prior to an accident; lagging indicators are metrics most commonly used to make comparisons based on established safety benchmarks of performance [19].

Using multi-leveled modeling, the authors of [20] examine empirical evidence of an association between leading indicators and safety performance. The results of their study support the impact of leading indicators as a means to eliminate or control the precursors of harmful incidents. The authors of [19] recognize the inherent difficulty in developing quantifiable indicators for some safety performance areas. Their work supports a broader approach to the use of LIs, which can be leveraged alongside the more traditional use of lagging indicators to better achieve organizational safety goals. Furthermore, the author of [21] notes that quantitative metrics alone did not provide an unbiased, clear picture of the overall safety culture of a site or project. They recommend that the statistical measurement of important variables such as near misses and unplanned shutdowns should be combined with a qualitative assessment of cultural variables such as accountability for safety, communication, integration of safety in all activities, open door policy, trust, management commitment, and learning attitudes. In construction safety, LIs can provide both quantitative and qualitative data.

\subsection{Proactive Worker Safety and Behavior}

While safety has always been a traditional research topic in construction, academic research on safety has been an increasing trend since 2012. Simulation and fall from height are emerging trends in construction safety studies that have received great attention in other research. Building Information Modeling (BIM) application and data mining techniques utilization are newer topics that attracted researchers to have more innovation in safety research topics [22]. Most of the research has been focused on accident prevention, risk assessment and hazard identification, safety behavior and evaluation of human factors in accidents. The safety research is being directed to study about the proactive measurements and maintain safety climate and culture on site [23]. Proactive safety is extremely important as it has been identified that the unsafe behavior of workers is the main cause of construction accidents $[24,25]$.

The authors of [24] utilize the agent-based modeling (ABM) approach to investigate the impact of workers interaction with their coworkers and management teams in interventions. They state that regular safety inspections and periodical safety training should be sustained in the construction process to ensure the safety of the jobsite. Management teams need to emphasize on leadership skills and high-level managers should also be involved in safety activities at the entirety of the project. The belief and support of senior managers have a great impact on safety goal achievement.

The authors of [26] state how demographic information and subgroup factors can affect safety planning. They described that human factors such as attitude, perception to safety, and individual behavior can impact safety planning and establish a safety climate on jobsites. They investigate workers from different demographic classes and report that: (1) workers' opinions have higher variation and less consistency in response and interpretation of the hazards that have a higher frequency but are less severe in jobsites; (2) younger and entry-level workers are more cautious and evaluate the hazards with a higher level of danger in comparison to the more experienced workers; (3) mid-level workers assume less danger for different hazards compared to the experienced, and entry-level workers; (4) 
interns evaluate hazards as more severe compared to workers. The authors of [26] suggest that safety training to be tailored based on the needs of different demographic categories.

The authors of [27] further study the effect of gender and education among Chinese workers and find that age and gender are significant to the recognition and perception of different hazards among workers where males had more confidence in confronting hazards which in other words means that they are more risk-takers. They also report that education level has a high correlation with the results of hazard recognition. The authors of [28] emphasize the importance of safety training and irregular inspection to the site for maintaining safety in jobsites and used an online platforms for visualization of safety audit results.

\subsection{Future Construction Work: Rise in Automation and IoT Technology}

Our society is currently witnessing the 4th Industrial Revolution, "which is blurring the separation of the physical, cyber, and biological worlds" [29]. Information and communication technologies (ICTs), such as the Internet of Things (IoT), Cyber-Physical-Social Systems (CPSS), Big Data, Artificial Intelligence (AI), and machine learning are starting to be adopted in the construction industry to ultimately increase the efficiency, safety, and quality of construction projects. "In a time when humans are increasing data creation every day, AI provides an endless resource for machines to learn and adapt ... The volume of data generated on an average construction site is growing-from images captured via mobile devices, to drone videos, security sensors, machine telematics, Building Information Modeling (BIM) and more" [30]. Data streams that have been received through IoT devices and sensor networks could be integrated with Building Information Modeling (BIM) tools and make a paradigm shift in construction efficiency improvement [31]. For example, automated speech and image recognition software is being developed commercially that utilizes AI and machine learning analytics for construction monitoring and control [32]. A user is able to define a "watchlist" of items for safety, quality and productivity, and the algorithms sift through a mass quantity of images and videos to identified problems and take swift action. What used to take multiple safety and project managers to ensure safety and productivity can all be done automatically in real-time. Robotics and automation are another emerging technology that may disrupt the construction industry. Robotics and automation have been prevalent in the manufacturing industry, and these practices are being adopted by the construction industry. The fusion of ICT and automation has given rise to industrialized construction (IC) by integrating manufacturing design and optimization tools for solving complex challenges in construction projects that can potentially increase economic benefits by aiming to reduce project delivery time, enhance quality control, and increase labor productivity. Assembly lines, prefabrication, and modular construction are revolutionizing how building components and houses are being constructed [33]. Robots have become such an emerging force that OSHA and a robotics industry trade group formed an alliance to ensure worker safety around robotics [34].

Currently, construction is heavily reliant on manual, time consuming, and labor-intensive processes. Technologies have improved some construction activities by assisting operators and workers. Sensing technologies, namely Global Positioning Systems (GPSs) and lasers, are enabling more precise and accurate earthwork operations, such as grading, hauling, scraping, and excavating. What once used to take many man-hours to measure and set up site lines can be fully automated with a push of a button. In addition to improving productivity, safety performance is another motivating factor for the adoption of automation in construction. Injury and accidents are all too common around heavy equipment. It is much safer if an operator is able to manage the project from a distance as it diminishes the chronic exposure to vibration, shock, and dust. Volvo Construction Equipment is developing an advanced warning system that sends a warning message to the operator to reduce the risk of accidents by utilizing AI algorithms to detect and decipher specific objects using several computer vision methods [30]. The use of automation can improve worker safety in construction environments by taking over some of the more dangerous tasks and allowing human workers to complete more 
cognitive tasks. Sensing and alert-based technologies, such as radio frequency identification (RFID), are being deployed to track and monitoring worker productivity and safety $[35,36]$.

\subsection{IoT for Construction Safety}

As part of the 4th Industrial Revolution, the construction industry is one of the most recent to implement the Internet of Things (IoT). The author of [37] presents a literature review on the Internet of Things (IoT) in high-risk Environment, Health and Safety (EHS) industries and identifies that one motivations of the adoption of IoT is to increase safety by the reduction of human error and disasters that lead to human and material casualties. Safety control is a significant issue in complex spatial environments such as airports and ports. IoT can help the geosimulation process to simulate the environment and assess workers' safety [38]. Since construction job sites are classified as high-risk work environments, [39] utilize an IoT architecture and propose an autonomous system for hazard recognition and sending safety notifications to site labors. The validation of system results shows that this new approach could be a cheap and effective system that has low operational costs, which supports the feasibility of their further utilization.

Sensors have helped in the development and advancement of safety studies. The authors of [40] comprehensively review the application of sensor technology in construction safety studies and emphasize the role of smartphones as a comprehensive sensor set which are always on a person. Smart phones' numerous advantages and ease of accessibility turns them into an optimal choice. The authors of [41] uses smartphones as IoT devices to recognize worker activities. The accelerometer and gyroscope sensors that have been embedded in smartphones are used to collect data and simulate human activities in floor reinforcing activities. The researchers successfully demonstrate the use of smartphones for this purpose and verify that prediction and classification of activities could be accomplished through smartphones.

Since the improper use of equipment can lead to accidents, the authors of [42] apply sensors and microcontrollers to recognize equipment activities to obtain feedback on the power consumption of different common equipment in construction sites such as hoist, electrical saw, and concrete mix machine. Through this process, they define different power consumption profiles, and the profiles could be used as a reference to monitor the equipment and check whether the equipment have been used correctly.

Roadway construction projects are one of the significant spaces with special work conditions as there are limited workspaces and dynamic hazard environment. Pedestrian workers are prone to being struck by the equipment, and the dynamic atmosphere will cause the location of potential hazards to be changed every day. The authors of [43] report that 609 fatal accidents have occurred in workers who were working in the close proximity of heavy equipment. Therefore, the authors of [43] implement Bluetooth technology for equipment proximity detection and safety analysis of roadway construction site and compared their result with the use of RFID technology. The researchers found that the use of Bluetooth is simple, requires minimum infrastructure and could reliably alert the workers which can add another layer of safety for avoiding the struck-by hazards. They also report that the Bluetooth systems require less calibration effort and yielded better results for capturing and transferring values. Bluetooth records also had less false-negative alerts, which adds to the reliability of Bluetooth systems. The system is also more robust in case of obstructions where a worker or research crew were making obstructions in line of sight. The authors of [44] developed "MapSafe" which is a cloud-based platform to enhance safety performance for infrastructure. The platform integrates Geographic Information System (GIS) and mobile technology with cloud computing atmosphere to capture, integrate, and visualize the safety data in infrastructure construction projects.

\subsubsection{Safety Recognition and Training}

The use of BIM is growing rapidly in the Architecture, Engineering, and Construction (AEC) industry and it is receiving considerable attention for safety practices, as it helps in early identification 
of potential hazards through the construction process. The authors of [45] state that safety planning is a requirement for the project lifecycle and BIM can be a useful tool for data management in $4 \mathrm{D}$ environment in this process. BIM provides a visual representation of the conflicts and potential hazards for their better understanding. Through an immersive environment, communication and collaboration will be highly boosted and this method could result in a significant saving in time and budget. The use of BIM in safety can also help in safety training and education.

The authors of [25] propose an image-based method for hazard identification and recognition of workers' unsafe behavior to help in early detection of potential hazards on site. The researchers use an innovative approach to analyze the infrared photos instead of regular photos to diminish the data-processing load.

The authors of [46] focus on crane operators and state that they are more vulnerable to human errors and tried to improve their situation awareness (SA) to help in reducing operators' level of error. They found that the assistance system can help the driver to improve their understandings from the surrounding and rendering decisions that are less risky.

The authors of [47] review a Real-Time Locating System (RTLS) for safety planning, and report that these systems have been used for safety monitoring and accident prevention, analysis of psychological status and ergonomics of workers, investigating safety behavior and transmitting timely safety warning for correcting unsafe behavior. The data that are captured through RTLS could also be used for hazard prediction, correction, and prevention. These systems could also help in real-time training of the workers and to gradually modify unsafe behaviors.

\subsubsection{Safety Monitoring}

The authors of [48] state that safety monitoring is a critical component to safety management and lack of effective communication between project stakeholders is a barrier to its enhancement. They propose a framework to help in the collection and analysis of safety information on-site to be disseminated to the stakeholders and help in communication. The main findings about the challenges and limitations in this development are as follow: (1) a proper risk level calculation method that can better quantify the risk rather than just using the severity score; (2) taking all the stakeholders' risks into consideration; and (3) the gradual adoption of these services.

Hoisting cranes has a high priority in safety planning in infrastructure construction, specifically for the construction of bridges and tunnels. To improve the quality of the safety monitoring process in infrastructure projects, the authors of [49] present a cyber-physical system for safety monitoring on a metro tunnel construction project in China. Internet of Things (IoT) guidelines are used to deploy wireless sensors and Wi-Fi for assisting in better control of the hoisting process. The researchers report the following challenges in their research: (1) hoisting environment in construction is complex; (2) blind spots and limited view of crane operator is a major problem; and (3) controlling the precision of cutter wheels in real-time is a complication.

\subsubsection{Wearable Technology}

Wearable technologies have become extremely popular over the past few years. These wearable sensors provide a comprehensive set of real-time data that will help in better understanding workers' behavior in accidents and hazardous situations. Workers' acceptance to wear these sensors is the first step in their implementation. The authors of [50] evaluate workers' opinion through an online survey and found that the companies' safety climate and workers' expected performance are two major factors in adoption of wearable sensors. They suggest that organizations who are interested in using wearable technologies to: (1) improve general workplace safety; (2) improve the safety atmosphere on site; (3) investigate practical ways to prove the applicability of wearable technologies for safety improvement on-site; (4) maintain an interactive and communicative environment between employees and employer to better illustrate the expectations and outcomes to the workers. 
The authors of [51] report that a major general contractor started to recruit IoT and wearable technologies for improving safety in construction sites and piloted a dynamic people tracking system labeled as Real-time Location System (RTLS). The system can collect and broadcast the accelerometer sensor information to identify falls and trips which can help in increasing the job site environmental awareness. The system can also monitor vital signs and identify the signs of heatstroke and fatigue and warn the workers and their supervisors about the detected signs.

The authors of [52] evaluate the use of wearable technologies and focus on smartwatches as these devices are becoming more common every day. After testing the smartwatches, the authors concluded that while they are affordable technology, they were not reliable devices for safety data acquisition.

\subsection{Summary}

The literature shows that IoT is an emerging trend for construction safety and has promising benefits that could improve construction safety. However, there has yet to be a comprehensive IoT-based system deployed in construction that can incorporate a multitude of heterogeneous sensors and devices. The current studies and applications only utilize a single system or technology. The authors of [40] discuss that systems that are based on single systems are not practical enough, but conversely, the complex sensor systems can yield satisfying results. Therefore, to advance the effectiveness of emerging technologies to achieve current challenges faced in the construction industry, there is a need for a comprehensive and interoperable IoT-based system that can be adaptable to the variety of sensors, technologies, and applications.

Due to the dynamic and time-sensitive nature of safety management's role in monitoring the safety process and worker behaviors, it is important for safety managers to actively monitor leading indicators and the state of safety in the workplace [53]. The authors of [54] state that the most effective leading indicators monitor the capacity of an organization to perform safely and drive the safety management system to continual improvement. Active Leading Indicators, as conceptualized as passive and active leading safety measures, have not been extensively researched at this point in time. There is limited information available regarding the use of passive or active leading indicators that best predict safety performance in the construction industry. In addition to providing much needed information regarding this emerging area of study, this research can help strengthen the case for the continued development and expanded field use of all types of leading indicators throughout the construction industry.

\section{Materials and Methods}

This paper is part of a larger researcher effort that involves design and evaluation of a secure IoT-based framework for construction safety and productivity. This research consists of two major components: (1) the interoperable IoT system framework for [55] and (2) an active leading indicator feedback loop. This research utilizes the prototype system IoT-ACRES [55], which is a distributed Software Defined Networking (SDN) managed and AI-assisted IoT-based system that can be adapted and extended based on needs of the research and application. The basic components include (1) smart objects, (2) local aggregators, (3) service gateways, and (4) cyber-secure Cloud networks (Figure 1). An in-depth discussion of the IoT-ACRES hardware and software is not in the scope of this paper.

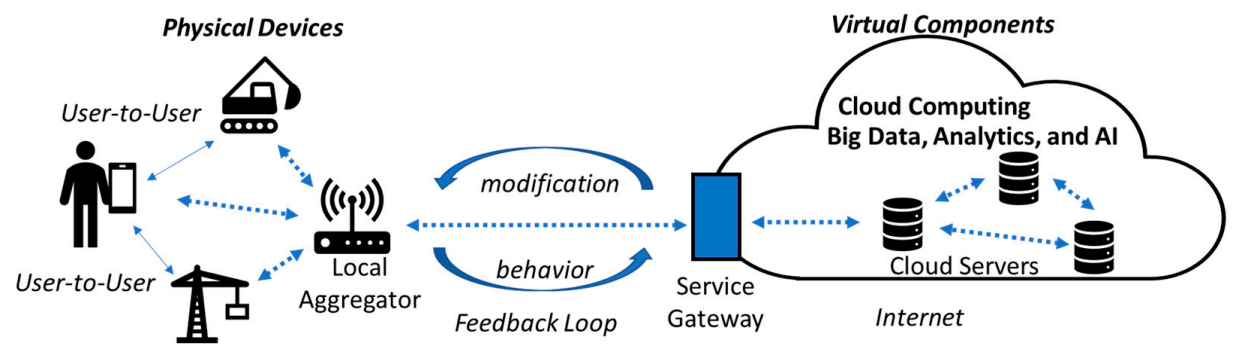

Figure 1. Internet of things (IoT)-ACRES cyberinfrastructure [55]. 
The physical devices and technology are a heterogeneous combination of devices, including RFID, wireless, and infrared communication devices, as well as sensors for electrical activity, proximity, motion, etc. Sometimes referred to as the "Internet of Things (IoT)" these devices represent the ability to have a multitude of heterogeneous devices communicate with each other without physical connections and can produce real-time data that can be used for analytics and optimization. Furthermore, these devices can remotely control robotic autonomous machines such as unmanned aircraft vehicles (UAVs), computer numerical control (CNC) machines, and 3D printers. Most notably, these systems include objects and sensors that can communicate over local aggregators, such as wireless local area networking (WiFi), Bluetooth, or Cellular (LTE) to connect to the internet to achieve various tasks. The virtual components are the cloud servers that host the various algorithms for Big Data, Analytics, and Artificial Intelligence (AI). A significant component of this network is the human and social aspect, which is critical for the feedback system to adjust functionality based on human behavior and preference. Furthermore, the "physical-virtual feedback" loop is essential to "understanding how the system can adapt to user preferences, how human behaviors can be modified to adapt to changing environments, and how the system can be modified for optimization" [56]. Therefore, the feedback loop is an essential and powerful tool to help improve human behaviors, in which the system can learn human behavior (e.g., record incidents, predict events), which in turns provides mechanisms of feedback to enable human behavior modification (e.g., alerts, reports). Significantly, humans and the system are continuously providing and receiving feedback that ultimately improves the system.

The data to be gathered for this study will utilize technology for quantitative measurement of leading indicators that effectively and significantly improve worker productivity and safety performance on construction projects. Additionally, this framework aims to establish and document the use of quantitatively measured active leading indicators in the construction industry.

Previous studies report that active leading indicators have been difficult to implement and evaluate due to the use of the subjective nature of the measures such as quality of job hazard analysis. This paper recognizes the previous limitations and seeks to create innovative active leading indicator measures that rely on technology rather than subjective evaluations.

The feedback loop in this research builds upon previous leading indicators research conducted by the authors of [7]. This model is derived from the Flowchart of Active Leading Indicators as reported by [6]. The feedback loop requires identification and evaluation of active leading indicators of safety performance. The scope of this research involves the development of a systematic framework for identifying salient leading indicators for specific types of organizations and projects and developing a tool that facilitates the use of leading indicators to evaluate safety risk and worker behaviors. This paper focuses on the development of quantitative IoT-enabled active leading indicator instruments. Data derived from these investigative instruments could then be coded and entered into analytical database for statistical queries and correlation of activities with performance indicators as well as related lagging measures of safety performance.

In addition to the ALI feedback loop, it is critical to identify and incorporate specific technology that will measure the process as well as provide tools for analysis and communication throughout the stream. Through the use of IoT-based technology, active leading indicator data can be gathered and utilized in a way that is clearly measurable and easily comparable. This paper aims to resolve the challenges faced during the previous active leading indicator study.

Specifically, the scope of this paper focuses on the development and implementation of the Active Leading Indicator Feedback Loop. The objectives are to identify leading indicators of value, methods of quantifiable data collection, means of timely analysis, communication of performance, and review of the effectiveness of the leading indicator to positively impact safety performance. The research methodology of creating an IoT-Based ALI system consists of 4 main steps: (1) identification of Active Leading Indicators and the data collection mechanism; (2) deployment of the IoT ALI data collection system; (3) implementation of the ALI feedback loop; (4) evaluation of the IoT ALI Data collection 
system and impact on safety performance. This study will demonstrate the practical application of technology and address the following research questions:

1. Are the devices gathering the data as intended?

2. Does the reported data have value?

3. How reliable are the IoT instruments?

4. How rugged are the IoT instruments?

5. What is the expected resource investment for an ALI system?

6. Is the ALI feedback loop functioning as planned?

7. Can safety management use the data in a timely manner?

8. Do management value the ALI system?

\subsection{Identification of Active Leading Indicators and Data Collection Mechanism}

The first step is to identify the inputs for the ALI Feedback Loop of active leading indicators, which requires the identification of a specific application needed to be monitored. Identification of the application requires a few considerations, such as the site conditions the workers will be exposed to, type of work performed, and any potential safety hazards that need to be monitored. Since there is a large variety of active leading indicators, the paper discusses three common safety incidents in construction: (1) equipment struck by; (2) slips, trips, and falls from height; and (3) physical stress by overexertion. Struck by (Indicator 1) and Falls (Indicator 2) are OSHA-identified Focus Four high-priority hazards [1]. Falls are the leading cause of death by workers in construction followed by struck-by incidents [57]. Physical stress/heat stress is an OSHA-identified hazard causing injuries and fatalities each year [58]. The following lists potential inputs for the three main active leading indicators.

\subsubsection{Active Leading Indicator 1: Monitoring for Struck-By Incidents}

Struck-by injuries are produced by forcible contact or impact between the injured person and an object or piece of equipment. Heavy equipment and motor vehicles are commonly involved in struck-by incidents. Potential data collection will be taken by proximity sensors placed on a minimum of four points. The purpose of these sensors is to detect impacts with equipment, materials, and other objects. Thresholds would be set to alert upon close proximity as well as impacts. Additionally, improper use of equipment is not always reported by workers. IoT equipment monitoring provides safety management with a new reliable data stream of equipment activity and operator behavior that can be monitored and reviewed. Equipment user behaviors can be analyzed for reward or correction based on behavior. Thresholds would be set to alert based on speed and sudden vector changes. Changes in behavior can be better detected and allow the safety staff to address safety breakdowns prior to a serious accident.

\subsubsection{Active Leading Indicator 2: Monitoring for Slip, Trip, and Fall Incidents}

Fall accidents are the number one cause of construction worker fatalities. Sensor-enabled wearables can help with this area by improving worker and site safety. It is proposed to use biometric data to monitor worker activity on the site. By gathering this information, sensor-based wearables could help prevent injuries and accidents, while also alerting safety management when slips, trips, and falls incidents occur. The sensors could also provide feedback such as a vibration when the worker approaches a fall hazard such as a leading edge or unprotected opening. This adds two-way communication to the workers as well as the safety staff. Thresholds would be established to alert based on proximity to fall points as well as sudden accelerometer changes due to slip/trips.

\subsubsection{Active Leading Indicators 3: Monitoring for Physiological Health Incidents}

The recognition of workers using excessive physical exertion is critical to the prevention of injuries and accidents at the construction site. Heart rate is regarded as a physiological indicator of worker 
exertion and fatigue. Sensor-based wearables are equipped with software to monitor and report real-time human biometrics such as heart rate, movement, temperature, and location. Safety staff can review all actively equipped workers through a computer pad or smart device. This information can be used by safety staff to manage individual as well as workforce exertion. Management equipped with information can better determine the use of rest/recovery periods to limit fatigue as well as attend to over-exerted workers. Monitoring will be ongoing for the duration of the shift. In general, a person's maximum heart rate is approximately 220 minus their age according to the American Heart Association. The specific threshold will alert managing staff when workers are exerting themselves at $90 \%$ or above for more than five min. Some sensor applications allow users to input age information that automatically calculates a heart rate percentage.

Based on the identified active leading indicators, Table 1 contains more detailed indicator information. For each ALI, there are specific applications, data inputs, and technologies listed. This table is designed to guide and track the application, data inputs, and specific use of technology for every indicator. As the ALIs are implemented, this table will be updated with additional information such as accuracy, reliability, and correlated safety impact. From this assessment, the researchers will report on performance, limitations and future recommendations for IoT-enabled active leading indicators.

Table 1. Major applications and technology for Active Leading Indicators (ALIs).

\begin{tabular}{|c|c|c|c|}
\hline ALI Monitoring & Application & Data Inputs & Potential Technology \\
\hline \multirow{5}{*}{$\begin{array}{l}\text { 1. Struck by type } \\
\text { incidents }\end{array}$} & 1.A.i Equipment Proximity & Proximity (closeness, striking) & $\begin{array}{l}\text { RFID, Ultrawide Band, GPS, Bluetooth, } \\
\text { Two-way hepatic feedback warning alert } \\
\text { when thresholds are exceeded }\end{array}$ \\
\hline & 1.A.ii Equipment Location & Location & RFID, Ultrawide Band, GPS, Blutooth \\
\hline & 1.B.i Worker Speed & $\begin{array}{l}\text { Operation of Equipment } \\
\text { (Speed) }\end{array}$ & GPS, Acceleraometer \\
\hline & 1.B.ii Worker Movement & $\begin{array}{l}\text { Operation of Movement } \\
\text { (sudden vector changes) }\end{array}$ & $\begin{array}{l}\text { RFID, Ultrawide Band, GPS, } \\
\text { Acceleraometer }\end{array}$ \\
\hline & 1.B.iii Worker Connection & Connection (seat belts/harness) & Bluetooth, Smart Watch \\
\hline \multirow{4}{*}{ 2. Slips, Trips and Falls } & $\begin{array}{l}\text { 2.A Anchor tie-off Personal } \\
\text { Fall Arrest System (PFAS) }\end{array}$ & Connection sensors & $\begin{array}{l}\text { Magnetic, Electrical Sensors, Two-way } \\
\text { hepatic feedback warning alert when } \\
\text { thresholds are exceeded. }\end{array}$ \\
\hline & 2.B Slips, Trips \& Falls (STF) & $\begin{array}{c}\text { Sudden vector changes, Body } \\
\text { Orientation }\end{array}$ & Wearables technologies, Accelerometer \\
\hline & 2.C Leading Edge & Proximity Location & $\begin{array}{l}\text { RFID, Ultrawide Band, GPS, Two-way } \\
\text { hepatic feedback warning alert when } \\
\text { thresholds are exceeded }\end{array}$ \\
\hline & 2.D Housekeeping & Location, Imaging & RFID, Bluetooth 360 Cameras, UAVs \\
\hline \multirow[t]{2}{*}{ 3. Physiological Health } & 3.A Worker Exertion & $\begin{array}{l}\text { Temperature, Heart rate, } \\
\text { Physiological status, } \\
\text { Ergonomics }\end{array}$ & $\begin{array}{l}\text { Wearables technologies, } \\
\text { Situation awareness monitoring, } \\
\text { Ultra-band enabled Wet Bulb enabled } \\
\text { temperature meter, ECG/EKG, Imaging }\end{array}$ \\
\hline & 3.B Site Safety & Location, Imaging & $\begin{array}{l}\text { Geographic Information System (GIS), } \\
\text { Infrared photos, Mobile Technology }\end{array}$ \\
\hline
\end{tabular}

\subsection{Deployment of IoT ALI Data Collection System}

The third step is to provide a proof of concept of the utilization of IoT to collect valuable data that can be utilized by safety staff. Although the technology framework is in the scope of this paper, it is important for the system to meet the needs and environmental conditions of the jobsites.

\subsection{Implementation of the ALI Feedback Loop}

The implementation of the ALI Feedback loop requires following steps 1-9 [7]: 
1. Identify the leading indicator

2. Define actionable metrics

3. Develop measurement process

4. Engage responsible parties

5. Implement

6. Analyze information

7. Communicate performance

8. Evaluate effectiveness

9. Recognition of successful indicators

\subsection{Evaluation of the IoT ALI Data Collection System and Impact on Safety Performance}

The fourth step is to measure and monitor traditional RIR rates of sites utilizing active leading indicators and compare the performance rates to similar projects that are not utilizing active leading indicators. Critical to the overall impact validation of the study is the measurement of the project recordable injury rate (PRIR). PRIR will be utilized as a benchmark to compare safety performance.

Through the utilization of a series of two-sample tests for each indicator, data analysis will indicate the active leading indicators that are associated with a lower project OSHA recordable injury rates. Project OSHA RIR (PRIR) values for projects that did not implement a specific indicator will be compared to the PRIR of the projects that did implement the indicator. Since the PRIRs are not normally distributed, a one-sided Wilcoxon Rank Sum test will be used to compare the sample means of PRIR and to compute a $p$-value.

The PRIR is a crucial data point which measures the safety performance of a project and is used as a comparison metric for the analysis. The purpose of this step is to measure the impact of active leading indicators. Considerations include whether IoT devices can produce leading indicators effectively and determining whether certain leading are indicators more effective than others and. This will be quantitatively measured by the PRIR rate. IoT-enabled LI sites should experience fewer accidents and as a result have a lower PRIR rate than non-IoT-enabled LI sites.

\section{Case Study}

As part of the initial validation of the ALI system framework, the researchers conducted a case study of a safety program utilizing heart-rate monitors to monitor worker exertion/stress. This study was approved by the University of Florida Institutional Review Board, study IRB201903189, on 10 December 2019. The site was located at a mine in the south-east section of New Mexico. Workers regularly conducted activities in a confined space in high ambient temperature (greater than $95^{\circ} \mathrm{F}$ ). This exposed the workers to a higher probability of an occurrence of a heat stress incident. There were approximately 1100 people on the case study site working in two ten-hour shifts (Shift 1 was 06:00-16:00 and Shift 2 was 15:00-01:00). The case site initially implemented personal heat stress monitoring of workers in May 2019. Each shift monitored approximately 30 people per shift based on work exposure.

Based on the sensitivity of the construction project, only basic information can be disclosed. During the preparation for implementation, workers expressed concern over privacy issues of the collected data. Additionally, workers expressed concern regarding the use of personal data that could reveal legally protected medical conditions. The safety program decided to focus on the real-time monitoring of worker stress with limited data collected other than determining whether the worker was experiencing excessive physical stress regardless of age, gender, and shift. The researchers recognized these issues as important challenges that will need to be addressed in future studies of implementation of personal worker data. 


\subsection{Identification of Active Leading Indicators and Data Collection Mechanism}

Mining operations occurred at a depth of over $2000 \mathrm{ft}$ below ground. The surface temperature of the site ranged from 90 to $100{ }^{\circ} \mathrm{F}$, with $15 \%$ relative humidity. The mine was ventilated through a system of motorized fans providing surface air to the underground work area. Working temperatures in the mine are typically higher due to restricted ventilation and worker/equipment activities. Higher humidity days also added to the discomfort of the mineworkers. Air quality within the mine was monitored by the Safety/IH program for hazardous levels of $\mathrm{O}_{2}, \mathrm{CO}_{2}, \mathrm{NO}_{2}, \mathrm{~S}$, as well as Volatile Organic Compounds (VOCs). The Active Leading Indicator identified in this study was 3A Physiological Heath-Worker Exertion.

The Safety/IH program purchased Polar H7 Bluetooth Smart Heart Rate monitors (Polar Electo Inc., Bethpage, NY, USA) as shown in Figure 2. The Polar H7 has the following features:

- Low-energy Bluetooth smart technology and coded $5 \mathrm{kHz}$ transmission, providing ECG-accurate heart rate to any Bluetooth smart ready device and compatible Polar training computers;

- Live heart rate on mobile training application, including the Polar Beat app;

- $\quad$ Long transmission range ( 10 m);

- Replaceable battery;

- Soft and comfortable hand washable fabric chest strap.

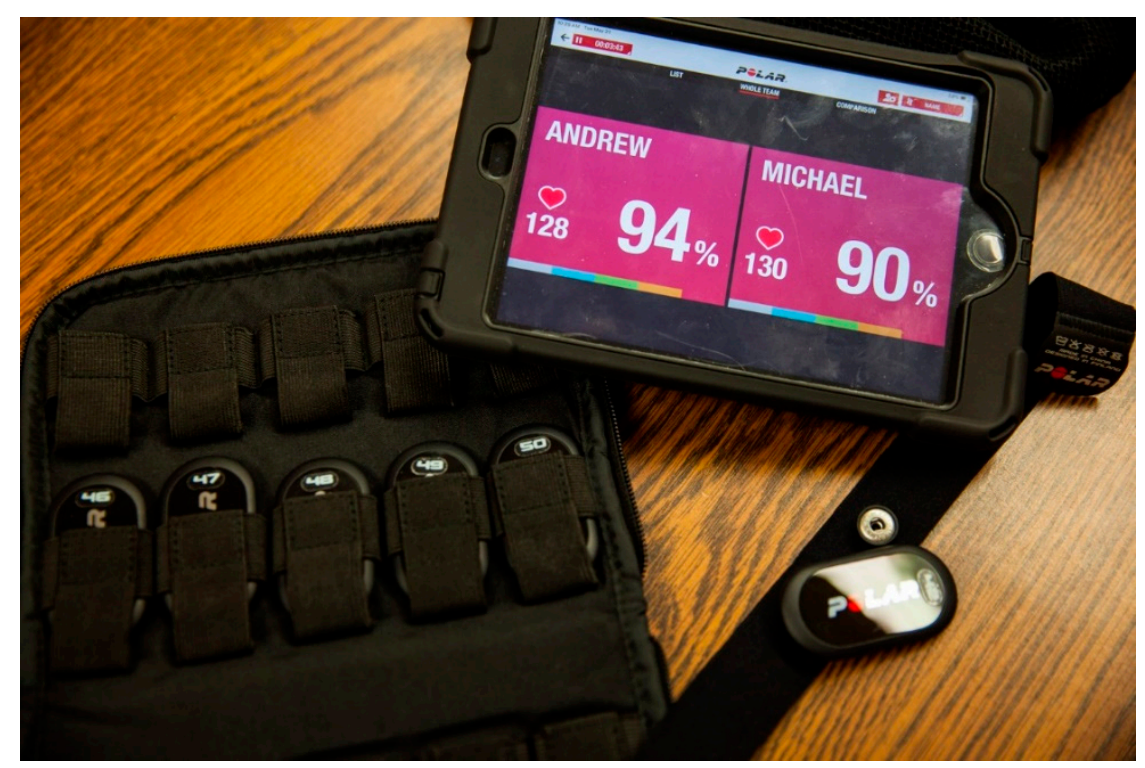

Figure 2. Onsite testing of the Polar Heart Monitor.

An important aspect of the Polar brand is it can connect to the IoT framework via the software development kit (SDK) and Polar Open AccessLink application programming interface (API). This enables the data to be accessed in real-time and used for analysis by any application that implements the API. This system included individual heart rate monitors with an elastic chest strap. Each worker wore a monitor under their clothes with the monitor positioned against the skin, over the heart area. These monitors cost approximately $\$ 50$ per unit.

As part of the monitoring of environmental site condition, the Safety/IH program purchased QUESTemp ${ }^{\circ} 36$ Area Heat Stress Monitors (TSI Incorporated, Shoreview, MN, USA) as seen in Figure 3. These monitors are designed to quickly and accurately evaluate site conditions. The WetBulb Globe Temperature (WBGT) is a measure of heat stress which takes into account temperature, humidity, wind speed, sun angle and cloud cover (solar radiation). This differs from the heat index, which takes into consideration temperature and humidity and is calculated for shady areas. Military agencies, 
OSHA and many industries use the WBGT as a guide to manage worker heat stress. Each unit costs approximately $\$ 3470$. The QUESTemp ${ }^{\circ}$ monitors provided the following data metrics:

- Dry bulb, wet bulb, and globe temperatures;

- Air velocity;

- WetBulb Globe Temperature (WBGT) (indoor index);

- WetBulb Globe Temperature (WBGT) (outdoor index);

- Heat index/Humidity Index (HUMIDEX);

- Temperature reading: Celsius or Fahrenheit;

- Time and date stamping with clock and calendar.

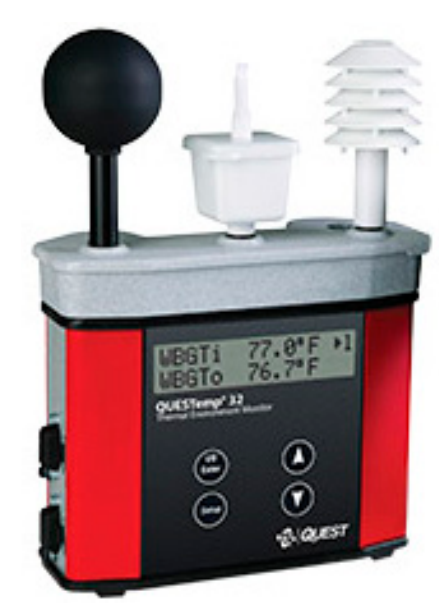

Figure 3. QUESTemp ${ }^{\circ} 36$ Area Heat Stress Monitors.

\subsection{Deployment of IoT ALI Data Collection System}

The project established an internet-enabled network around the site. Due to the sensitivity of the project, there was high security which prohibited direct access from the local devices to the global internet. Referring to Figure 1, this means that the "service gateway" was essentially blocked due to high monitoring (only certain information was able to obtain access). However, the site still had a local server network, the local aggregators still had connectivity (e.g., Bluetooth), and the process and feedback loop were conducted on the local server (e.g., iPad).

Each worker on shift was given a Polar heart rate monitor. Heart rate information was transmitted wirelessly via Bluetooth to pad-sized computers running the default Polar proprietary software. From the pad, a supervisor could actively monitor and track the exertion of each worker. The software allows the supervisor to track all members of the team wearing the devices. Utilizing this real-time data stream, supervisors and safety staff could identify workers at risk of overexertion and respond prior to a safety incident. Once a rested worker had attained sufficient recovery, the safety staff would direct the worker to resume duties. Thresholds were established to alert managing staff when workers are exerting themselves at $90 \%$ or above for more than five min. The wearable technology is an enhancement to traditional safety monitoring techniques such as worker observation and direct safety personnel supervision. The wearable technology is implemented to enhance safety management and provide an additional set of data for safety staff to utilize to best manage work practices.

In total, 10 QUESTemp ${ }^{\circ} 36$ Area Heat Stress Monitors were placed at various locations around the site in proximity to worker activity-five were placed above ground and five were placed below ground. Due to technical limitations, these systems were not wireless and could not connect the to the local network as part of the IoT system. 


\subsection{Identification of Active Leading Indicators and Data Collection Mechanism}

\subsubsection{Step 1-Select the Active Leading Indicator}

Project management identified heat stress and heart rate as active leading indicators. These indicators were chosen for several reasons. First, this site previously experienced serious worker incidents due to heat stress and overexertion. Second, the work site is located in a desert climate experiencing high temperatures creating an environmental hazard. Third, many workers on site are required to work in specialized Personal Protective Equipment (PPE) that significantly raises body temperatures.

\subsubsection{Step 2-Define Actionable Metrics}

Heart rate was identified as a physiological indicator of worker exertion and fatigue. Safety staff examined information from the American Heart Association and created a metric to alert safety staff of workers meeting actionable levels. A person's maximum heart rate is generally approximately 220 minus the age of the worker. The specific threshold will alert via text and color on the Bluetooth-connected pad monitor when a worker exerts themselves at $90 \%$ or above for more than five min.

The specific threshold will alert managing staff when workers are exerting themselves at $90 \%$ or above for more than five min. Some sensor applications allow users to input age information that automatically calculates a heart rate percentage. Sensor software applications allow users to input age information that automatically calculates a heart rate percentage. Site safety management developed an alphabetical code system (O A B C D E) to identify and communicate work protocols based on the site measured WBGT, see Table 2. This metric table was printed on laminated cards and distributed to all workers to hang on their work badge lanyard. For each WBGT category, there is a category for work/rest min per hour based on duty level (light-very heavy). The colors on the table serve as a visual aid for quick interpretation of the work hazard. The colors indicate the severity of the work conditions to determine what safety measures are needed, in which green indicated safe (no restrictions), yellow means caution (work/rest restriction), and orange means severe (physiological monitoring devices are required).

Table 2. WetBulb Globe Temperature (WBGT) work/rest Guide.

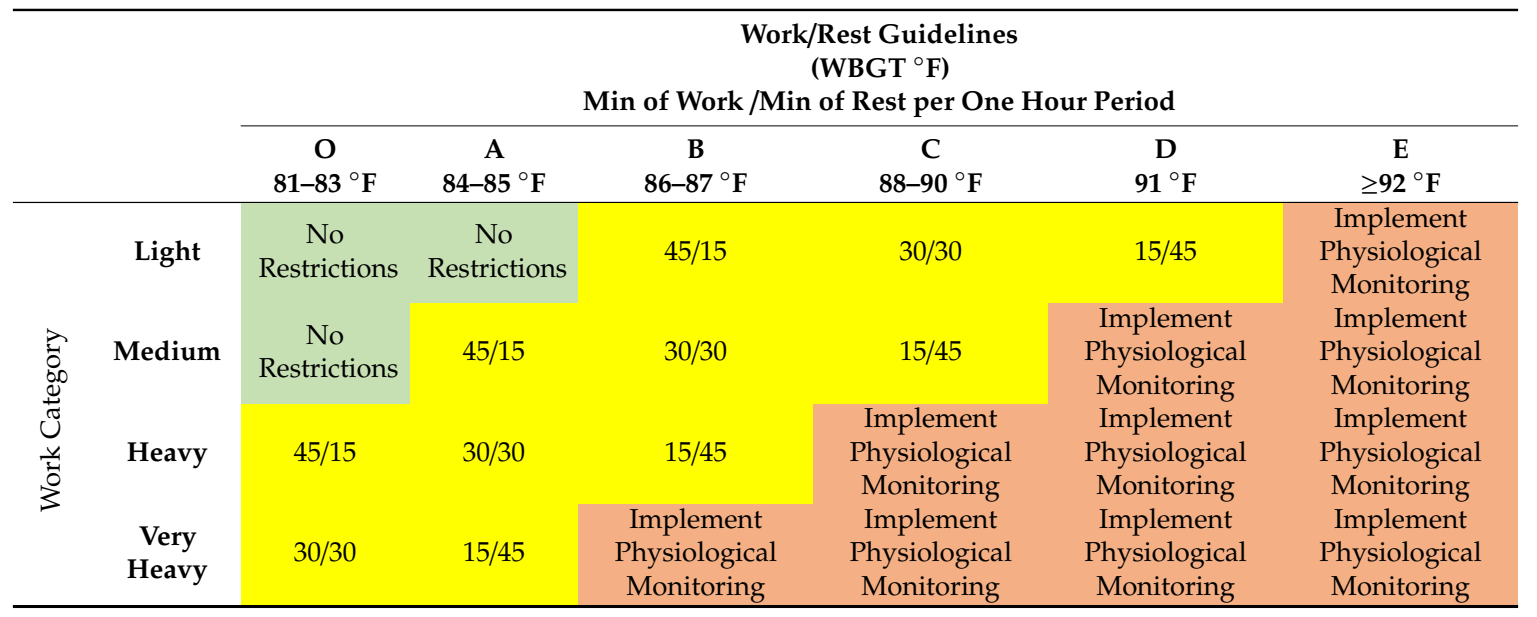

Criteria is for workers in normal work clothing unless adjustments are made for PPE; WBGT is measured in degrees Fahrenheit and combines the effects of radiant heat, humidity, and air movement; It is not necessary to leave the area to take a break; pausing work activities is sufficient; Work conditions color code: green means safe, yellow means work/rest restrictions, and orange means physiological monitoring devices are required. 
Additionally, the safety team created temperature adjustments based on the type of worker PPE, Table 3. The WBGT was monitored hourly by and recorded by hand. Updates of the site work status were communicated via radio and PA systems from a central monitoring station.

Table 3. Personal Protective Equipment (PPE) adjustments based on temperature.

\begin{tabular}{cc}
\hline Personal Protective Equipment (PPE) & Adjustment to Temperature Values \\
\hline One pair OREX Original (with hood) & add $5^{\circ} \mathrm{F}$ \\
Level 3 ARC Flash Protection & add $6{ }^{\circ} \mathrm{F}$ \\
One pair OREX Ultra (with hood) & add $11^{\circ} \mathrm{F}$ \\
One pair OREX Original and One Pair OREXUltra & Consult IH-Phsiological Monitoring Required \\
Two Pair OREX Ultra (with hood) & Consult IH-Phsiological Monitoring Required \\
Any Encapsulated Suit Apparel & Consult IH-Phsiological Monitoring Required \\
Level B PPE or Firefighter Turnout Gear & Consult IH \\
ProForce Body Armor & Consult IH \\
\hline
\end{tabular}

\subsubsection{Step 3-Develop Measurement Process}

Heart rate monitoring was conducted by the Polar H7 Bluetooth Smart Heart Rate Monitor. Workers wore monitors that measured heart rate via skin sensors. Data was collected and sent via Bluetooth to supervisors using pad computers. Software calculated worker heat rate and displayed visual alerts for people exerting at $90 \%$ or above for more than $5 \mathrm{~min}$. The process for environmental temperature monitoring involves utilizing the QUESTemp ${ }^{\circ} 36$ Area Heat Stress Monitors. Since these sensors are not networked, safety staff were required to conduct readings and input them into an hourly log. This process would greatly benefit from real-time IoT connectivity.

\subsubsection{Step 4-Engage Responsible Parties}

The Safety/IH programs developed active leading indicators with guidance from owner representatives, management as well as labor representatives. This program also received guidance from the Voluntary Protection Program (VPP) Committee safety representing all areas of the company. For example, the VPP committee recommended the creation of the laminated guide cards as a way to better communicate the function and relevancy of Heat Stress Monitoring program.

\subsubsection{Step 5-Implement}

In May 2019, the site implemented Environmental Temperature Monitoring and Heart Rate Monitoring. The Safety/IH program timed the implementation of these active leading indicators as a means to mitigate the hazards of working in the hot summer climate.

\subsubsection{Step 6-Analyze Information}

Information was gathered for Environmental Temperature and Heart Rate Monitoring. The data was used by the Safety/IH program to respond to changes in environmental and worker conditions in a timely manner. In regard to short term data, the ALIs provided the safety program with quantifiable and easy to understand prompts to guide the workforce. Regarding long term data analysis, the researcher's duration with the case study was three months. At the end of the case study, the Safety/IH staff were still analyzing the data of these two new leading indicators.

\subsubsection{Step 7-Publicize Performance}

The Safety/IH program promoted the new Heat Stress and Heart Monitoring programs. These were communicated company wide. The VPP Committee recognized the efforts of the new programs, providing valuable input to help workers understand the benefits of Heat Stress program. 


\subsubsection{Step 8-Evaluate Effectiveness}

At the end of the case study, the Safety/IH staff were still evaluating the effectiveness of these two new leading indicators. These leading indicators were developed as a means to resolve mistakes of the past and prevent recordable injuries due to heat stress and overexertion. For the period May 2019 through August 2019, there were no heat stress or exertion-related recordable incidents. This was an improvement from previous year's performance for the given period of time of the year. This resulted in a significant improvement of the site recordable injury rate. More details are discussed in the Results and Findings Sections.

\subsubsection{Step 9-Celebrate}

Through formal memos, emails, and the company web page, top management announced several safety milestones accomplished for the period May 2019 through August 2019. In particular, management focused attention on congratulating the workers on achieving significant improvements in measured safety performance.

\section{Results and Findings}

Given the heat stress condition of working in a mine, the use of the Polar Heat monitors provided the safety staff with more accurate information to assess the health risks of the workers. This is particularly important given the diversity of the workforce in terms of age, weight, and fitness. Safety staff found this a valuable tool to schedule work breaks for individuals as well as the entire crew. The answers to research questions of this study are shown in Table 4.

Table 4. Research questions/results.

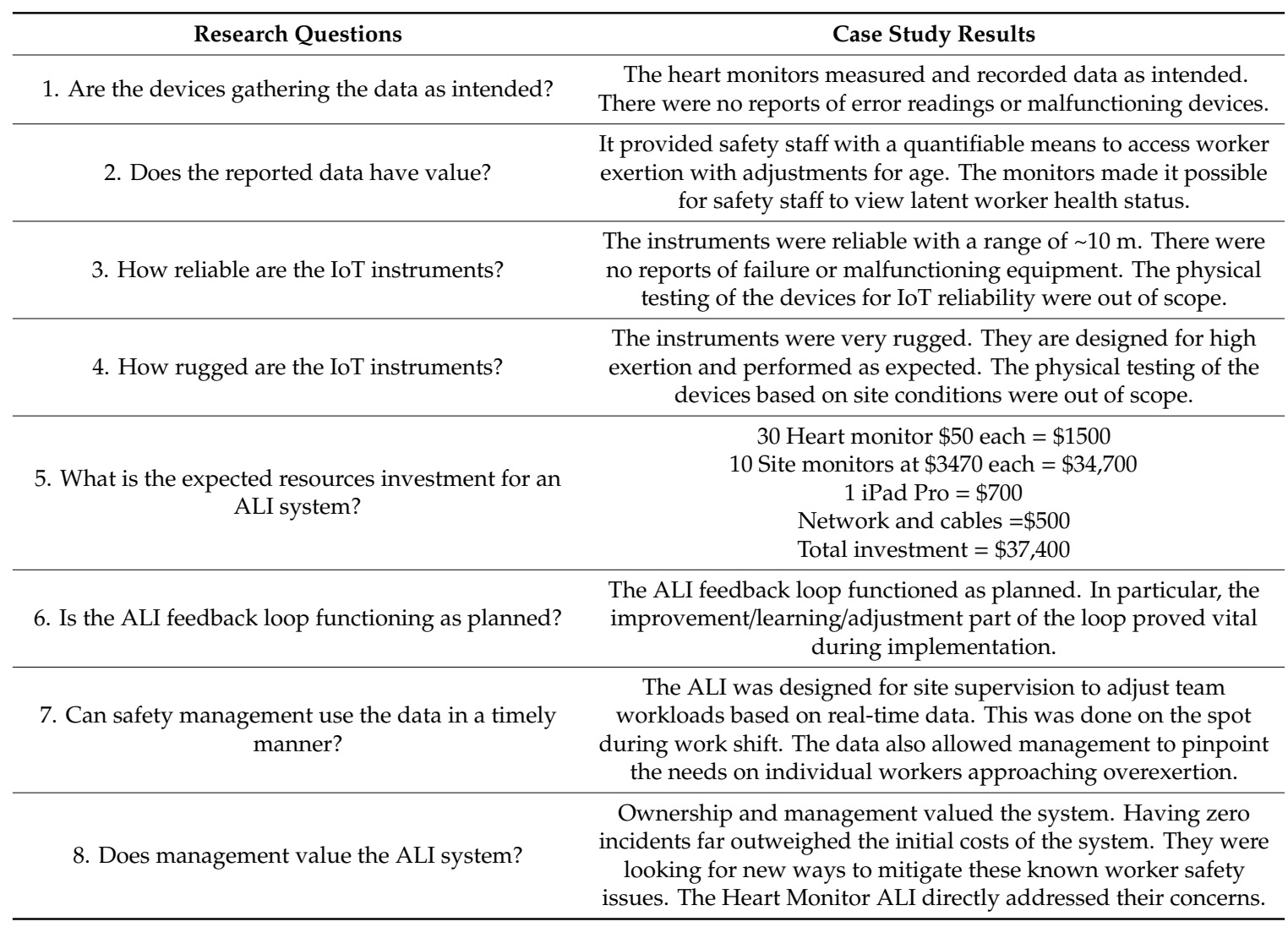




\subsection{Worker Acceptance: Push back}

Workers were generally positive regarding the use of the monitors. Fitting was easy and the workers did not find the monitors cumbersome to their activities. However, some workers voiced concern over the privacy of the personal health information that was gathered.

\subsection{Non-Disclosure of Medical Conditions}

After a period of time, it was apparent that the heart monitors could possibly identify specific people with certain medical conditions such as high blood pressure. People who were more often identified as at-risk by the monitors typically had medical conditions such as excessive weight or diabetes. With the development of more accurate health monitors, safety programs will have to develop standards of practice that allow for safe work behavior while respecting and providing confidentiality regarding a worker's medical history. Some workers voiced concern that the monitors may be used by management in a way that would preclude them from working in the future because of their medical condition(s). These monitors have strong future implications for the determination of worker fitness for duty.

\section{Considerations for the Next Testing}

Due to the constraints of the mine, a real-time internet connection was not feasible. Based on the study, it would be more useful to connect the heart monitors to an internet-enabled network that can allow for greater physical range of monitoring. During the case study, Safety/IH had to manually upload data from the devices to the internet. This takes up the valuable time of safety professionals as well as slows down the analysis process. Additionally, the information gathered could be better utilized if it was recorded on the network in addition to the site device. Even in the mine, a local network system could be deployed that can capture and store the data in real time and will only be collected or sent to the Safety/IH on less frequent intervals.

\section{Discussion}

\subsection{IoT Produced Active Leading Indicator Data}

As previously discussed, there has been a lack of technology to produce the leading indicator that is quantifiable and measurable to be considered active. This study demonstrates that IoT-enabled technology does have the capability of producing quantifiable data that can trigger an actionable response in real-time based on established thresholds when properly designed and implemented. In the case study, workers were monitored for heat stress working in a mine where temperatures were relatively consistent. In the field of surface construction, the use of such monitors could have an even greater impact due to the dynamic heat conditions experienced on site. The use of heart monitoring devices at the case study site provided safety staff with monitored worker health information that was not available in the past. During the previous year, a worker suffered a serious recordable heat stress incident that resulted in hospitalization. In hindsight, this worker injury could have been avoided if the worker was utilizing the Polar Heart monitor. The safety staff developed this new safety monitoring program to prevent an accident from reoccurring. During the researchers' time at the case study, there were no worker recordable incidents involving heart or heat stress. Based on the performance, the safety program at the case study site will expand the use of the heart monitoring program.

\subsection{Risks of Automation and Technology in Construction}

With the rise of automation and technology in construction to increase productivity and safety, there is a growing risk that valuable jobs will be lost, especially skilled and unskilled labor. According to a recent study conducted by the Pew Research Center, $72 \%$ of Americans are worried that automation will overtake jobs and $42 \%$ think it will disrupt the construction industry [59] Additionally, the increase 
in safety standards and the high cost of safety mitigation to meet standards, such as OSHA's safety requirements, will create market pressures for construction companies to offset the direct and indirect costs of worker exposure to hazardous building materials. The adoption of technology is inevitable; however, it is possible to augment human performance to develop new skills and work in tandem with technology. Therefore, is important understand and advance the human-technology partnership by training workers to utilize technologies to increase quality, productivity, and safety in construction: the goals that have resulted in the rise of automation in construction.

\subsection{Symbiotic Relationship: Augmentation of Human Behavior and Technology}

If successfully implemented, the automation of IoT-based construction will ultimately shape the future of work in construction. Therefore, it is imperative that the current workforce adapt and create a symbiotic relationship with the emerging technology. However, worker acceptance to emerging technology, especially intrusive technologies that can track and monitor a worker, is extremely limited. As seen in the present case study and previous studies [35,36], there has been extreme pushback for user acceptance. As the many challenges faced by the construction industry continue to grow, technology will play an integral role in supporting the current workforce. The augmentation of technology will continue to educate and inspire the next generation of workers. Technologies can increase worker efficiency and can even preserve jobs by helping companies improve efficiencies, reduce costs, and increase value. Technology will play an integral part within the construction industry, and it is vital to augment human behavior to utilize these technologies, ultimately giving companies the advantages of automation while resolving the shortage of laborers and the growing demands of commercial construction and future work.

\subsection{Incident of Concern (IOC)}

Recording safety incidents is a sensitive activity because of negative repercussions. Many safety incidents that are not required by law to be reported, such as near misses, are often under reported [60]. The authors of [61] group four major reasons for not reporting safety incidents: (1) fear of either disciplinary action or embarrassment; (2) risk acceptance due to the mindset that incidents are part of the job and cannot be prevented; (3) useless to report since it may be perceived that management will not act on the situation; (4) practical reasons such as being inconvenient or time consuming. For example, contractors and workers alike are hesitant to report incidents, especially near misses, because they may feel like their reputation is at stake as well as reducing any fines paid for violations. However, [62] conclude that by encouraging workers to report incidents, employees perceive the costs associated with accidents as being high and thus reduce the chances of future accidents. Therefore, in order to encourage reporting and alleviate the negative perceptions of near misses, we propose the concept of Incident of Concern (IOC).

An IOC is defined as an incident or occurrence on site that did not result in an injury or fatality despite the existing potential. While no one wants to experience a potentially harmful event, it is important to note the value of collecting information surrounding an IOC. This data has tremendous usefulness to safety management, especially when preventing future accidents. For example, by utilizing Active Leading Indicator 1: Equipment monitoring for struck-by incidents, safety staff will be informed of all proximity contact made by monitored equipment. Some of these recorded events will not result in injury and qualify as a recordable incident. Despite this being an unwanted event, the fact is the event did occur and fortunately was reported through the ALI system. Safety management is in a better position by knowing about this IOC. With this information, the safety program can correct and prevent future occurrences that certainly could lead to an injury or fatality without intervention.

It is vitally important for safety personnel to utilize data from an IOC in a proactive way. Tracking and using IOC information creates data streams, prompting the attention of safety management to better recognize and respond to breakdowns in the safety program prior to the occurrence of an actual accident. Discouraging or disregarding IOC information creates blind spots and prevents safety 
management from accurately assessing the status of the safety of the site. In fact, the active leading indicators produced by the IoT system remove the personal and subjectivity of the data. By doing so, the ALIs will identify behaviors that can be remediated.

\section{Limitations and Barriers}

This study includes a few limitations and potential drawbacks that need to be considered in future research. The main limitation of the study is due to worker privacy concerns, legal issues, and project sensitivity, only certain information is able to be collected and discussed. Therefore, certain analysis and assessments are not included. Future studies with proper authorizations to disclose personal information are required to report more scientific results, such as worker demographics, worker safety records, and assessments on the system's impact on the safety performance.

The second limitation is that the study only focuses on one of the many safety incidents. However, the intent of this study is to validate the feasibility of the CII active leading indicator framework with IoT systems, which has been deemed successful. Future research will include other leading indicator systems.

The third limitation is due to the site being a secure mine in the desert-the study was not able to obtain access with a direct connection to the internet. Although this did not affect the study since the site has a closed local area network, having a true internet-based system could enable the connection to other sources of real-time information, such as weather. An internet-based connection can streamline data access and reporting. Having limited access sites, such as an underground mine or remote location, would require the use of hybrid connection devices to relay information from the site local access points to a central server that has a connection to an internet service provider.

Other limitations include site contracts, which included a sensitive and secure mining operation. A major recommendation for future research is to establish a safety training program that educates workers on IoT-based systems and discloses the importance of the system. Utilizing Incidents of Concern (IOC) is another recommendation that can enable more transparency and acceptance of the system. Furthermore, limitations of the hardware and safety devices, such as battery power and signal strength, have not been considered. This case study used manual human observers (i.e., safety managers) to ultimate observe worker safety. Future research to test the physical limitations and reliability of the hardware is required.

Finally, potential unintended consequences for using IoT sensors need to be recognized. The authors of [63] report that the following unintended consequences must be considered when adopting the use of IoT-enabled wearable sensors:

1. Unintended modification of behavior;

2. Unintended creation of big datasets, and repercussions of their use and misuse;

3. Unintended privacy and security issues which are specific to wearable devices;

4. Unanticipated challenges facing regulatory bodies, which must somehow regulate both the safety and security of wearable devices and associated apps which interpret the acquired data.

Informed by the experience and challenges from the case study, future implementation of IoT-based leading indicators must examine these important factors as an integral part of the study.

\section{Conclusions}

Maintaining a high level of safety is of the utmost importance for the well-being of construction workers. Traditionally, safety performance in the construction industry has been measured by historical information about some aspect of the measures of safety performance that has occurred, known as lagging indicators. Lagging indicators may not be the most effective means to prevent future occurrences of accidents and injuries over the entire course of a given project in light of the rapid development of new technologies for safety and worker behavior measurement. In contrast, leading indicators (LIs) are measures that can be used as predictors of future levels of safety performance 
because they reflect information about the safety process prior to the occurrence of a safety incident. Specifically, active leading indicators (ALIs) have the potential to identify safety hazards and take immediate actions to prevent incidents. However, there is a major gap in research that incorporates a fully automated ALI system. Therefore, this paper addresses this gap by presenting a new method that utilizes the Internet of Things (IoT) to collect quantifiable data that can trigger an actionable response in real-time based on established thresholds. This novel method integrates the Construction Industry Institute (CII) active leading indicator framework with the prototype IoT-ACRES system. Significantly, the ALI provides a human-technology feedback loop, which is the essential aspect of the IoT system because it provides real-time feedback to both the users and systems.

A case study was presented to validate the IoT-based ALI framework. Bluetooth-enabled heart rate monitors were issued to workers in a hazardous and critical mining construction site. The ALIs that were recorded included heart rate and body temperature. Thresholds were established that alerted the safety manager when a worker exhibited potentially unsafe conditions. The results of the study demonstrated the feasibility of the system. Additionally, other insights that were revealed include: worker resistance; non-disclosing of medical conditions, and limitations for IoT connectivity. The originality of this research is that it the first to integrate active leading indicators with IoT technology in construction. This paper contains the following contributions:

\section{(1) Novel IoT-based human-technology feedback loop for worker safety}

Although IoT-based systems are being deployed for construction applications, there is a gap in human-technology feedback. This feedback loop is an important aspect that brings the "smartness" to devices, since Big Data, analytics, and artificial intelligence (AI) techniques can be utilized to learn user habits. The human and social dimension is critical for the feedback system to adjust functionality based on human behavior and preference. Therefore, this loop works in real time, where the user can obtain immediate feedback (e.g., alerts), while the system can record user habits to modify the system (leading indicators). This paper integrates the CII ALI framework directly into the loop, where the system can learn the worker behaviors to produce active leading indicators.

(2) Demonstration of the CII active leading indicator framework with IoT systems to enable active leading indicators

Previous research by CII developed an active leading indicator framework to prevent safety incidents. However, due to the limitation of technology, the framework could not be implemented for validation. Therefore, this paper utilizes emerging IoT-enabled technology to provide real-time data needed for the framework. A case study that utilized Bluetooth-enabled heart rate monitors was used to demonstrate the ALI framework.

(3) Identification of the potential inputs to the ALI framework from emerging IoT-enabled systems

Since each safety incident has different variables for measuring and predicting safety incidents, it is important to identify what data is needed. Therefore, this paper presents potential inputs and potential technology that can be provided by IoT-based systems for the three major occurring incidents: (1) equipment struck by; (2) slips, trips, and falls; and (3) physiological health.

(4) Development of the concept of Incident of Concern (IOC)

Due to the hesitation of reporting safety incidents, this paper proposes the concept of Incident of Concern (IOC), where incidents, including near misses, can be recorded without any negative consequences. An IOC is defined as an incident or occurrence on site that did not result in an injury or fatality despite the existing potential. While no one wants to experience a potentially harmful event, it is important to note the value of collecting information surrounding an IOC. This data has tremendous usefulness to safety management. The IoT-based ALI framework identifies and produces data that can be used without personally identifying the culprit. 
Active Leading Indicators have shown the potential for modifying human behavior to prevent safety incidents. Our industry is seeing a rise in IoT applications, enabling a safer and smarter construction site. The broader impact of this study is that the ALI framework can be incorporated into the emerging IoT applications to enhance their safety impact. Significantly, incorporating an IoT-based system that utilizes the CII ALI framework holds great potential to eliminate many of the safety incidents and fatalities that occur in the construction industry. Furthermore, the data produced by the system can be used in deep-learning algorithms to identify trends and make forecasts to further enhance the prevention of safety incidents.

Author Contributions: Conceptualization, A.C. and A.W.; methodology, A.C. and A.W.; software, A.C.; validation, A.C. and A.W., formal analysis, A.C. and A.W.; investigation, A.C., A.W., and A.A.; resources, A.C., A.W., and A.A.; data curation, A.C. and A.W.; writing-original draft preparation, A.C., A.W., and A.A.; writing-review and editing, A.C., A.W., and A.A.; visualization, A.C. and A.W.; supervision, A.C.; project administration, A.C. and A.W.

Funding: This research received no external funding.

Conflicts of Interest: The authors declare no conflict of interest.

\section{References}

1. Bureau of Labor Statistics (BLS). Economic News Release. Table 3. Fatal Occupational Injuries Counts and Rates for Selected Occupations, 2016-2017. Available online: https://www.bls.gov/news.release/cfoi.t03.htm (accessed on 8 June 2019).

2. NBC 4 New York. Air Conditioning Unit Plunges Nearly 30 Stories in Midtown Crane Accident. 10 Hurt. 2015. Available online: https:/www.nbcnewyork.com/news/local/Crane-Collapses-in-Midtown-Manhattan305606991.html (accessed on 25 February 2018).

3. Bureau of Labor. Focus on Productivity: Construction Industries. 2018. Available online: https://www.bls. gov/lpc/construction.htm (accessed on 15 May 2018).

4. OSHA. OSHA's \$afety Pays Program. United States Department of Labor, 2015. Available online: https: //www.osha.gov/dcsp/smallbusiness/safetypays/estimator.html (accessed on 18 May 2018).

5. Hinze, J.; Thurman, S.; Wehle, A. Leading Indicators of Construction Safety Performance. Saf. Sci. 2013, 51, 23-28. [CrossRef]

6. Hallowell, M.; Hinze, J.; Baud, K.; Wehle, A. Proactive Construction Safety Control: Measuring, Monitoring, and Responding to Safety Leading Indicators. J. Constr. Eng. Manag. 2013, 139, 04013010. [CrossRef]

7. Construction Industry Institute (CII). Implementing Active Leading Indicators; Implementation Resource 284-2; University of Texas at Austin: Austin, TX, USA, 2012.

8. Cooper, D. Management Safety Culture, A Model For Understanding \& Quantifying A Difficult Concept. Prof. Saf. 2002, 47, 30-36.

9. Wamuziri, S. Framework for Change in Safety Culture in UK Construction. Evolution of and Directions in Construction Safety and Health. In Proceeding of the CIB W99 International Conference, 14th Rinker International Conference, Gainesville, FL, USA, 9-11 March 2008; pp. 193-207.

10. Laurence, D. Safety Rules and Regulations on Mine Sites-The Problem and a Solution. J. Saf. Res. 2005, 36, 39-50. [CrossRef]

11. Colley, S.; Lincolne, J.; Neal, A. An examination of the relationship amongst profiles of perceived organizational values, safety climate and safety outcomes. Saf. Sci. 2013, 51, 69-76. [CrossRef]

12. Christian, M.S.; Bradley, J.C.; Wallace, J.C.; Burke, M.J. Workplace safety: A meta-analysis of the roles of person and situation factors. J. Appl. Psychol. 2009, 94, 1103. [CrossRef]

13. Clarke, S. The relationship between safety climate and safety performance: A meta-analytic review. J. Occup. Health Psychol. 2016, 11, 315-327. [CrossRef]

14. Nahrgang, J.D.; Morgeson, F.P.; Hofmann, D.A. Safety at work: A meta-analytic investigation of the link between job demands, job resources, burnout, engagement, and safety outcomes. J. Appl. Psychol. 2011, 96, 71-94. [CrossRef]

15. Wurzelbacher, S.; Jin, Y. A framework for evaluating OSH program effectiveness using leading and trailing metrics. J. Saf. Res. 2011, 42, 199-207. [CrossRef] 
16. Toellner, J. Improving Safety \& Health Performance: Identifying \& Measuring Leading Indicators. Prof. Saf. 2011, 46, 42-47.

17. Grabowski, M.; Ayyalasomayajula, P.; Merrick, J.; McCafferty, D. Accident Precursors and Safety Nets: Leading Indicators of Tanker Operations Safety. Marit. Policy Manag. 2007, 34, 405-425. [CrossRef]

18. Sinelnikov, S.; Inouye, J.; Kerper, S. Using leading indicators to measure occupational health and safety performance. Saf. Sci. 2015, 72, 240-248. [CrossRef]

19. Persson, K.D.; Lederman, L.; Palomo, J.; Szikszai, T. Safety Performance Indicators. In Proceedings of the International Conference on Topical Issues in Nuclear Safety, Vienna, Austria, 3-6 September 2001; pp. 271-290.

20. Sheehan, C.; Donohue, R.; Shea, T.; Cooper, B.; De Cieri, H. Leading and lagging indicators of occupational health and safety: The moderating role of safety leadership. Accid. Anal. Prev. 2016, 92, 130-138. [CrossRef] [PubMed]

21. Mengolini, A.; Debarberis, L. Effectiveness Evaluation Methodology for Safety Processes to Enhance Organizational Culture in Hazardous Installations. J. Hazard. Mater. 2008, 155, 243-252. [CrossRef]

22. Costin, A.; Adibfar, A.; Hu, H.; Chen, S. Building Information Modeling (BIM) for transportation infrastructure-Literature review, applications, challenges, and recommendations. Autom. Constr. 2018, 94, 257-281. [CrossRef]

23. Jin, R.; Zou, P.X.; Piroozfar, P.; Wood, H.; Yang, Y.; Yan, L.; Han, Y. A science mapping approach based review of construction safety research. Saf. Sci. 2019, 113, 285-297. [CrossRef]

24. Zhang, P.; Li, N.; Jiang, Z.; Fang, D.; Anumba, C.J. An agent-based modeling approach for understanding the effect of worker-management interactions on construction workers' safety-related behaviors. Autom. Constr. 2019, 97, 29-43. [CrossRef]

25. Yu, Y.; Guo, H.; Ding, Q.; Li, H.; Skitmore, M. An experimental study of real-time identification of construction workers' unsafe behaviors. Autom. Constr. 2017, 82, 193-206. [CrossRef]

26. Han, Y.; Feng, Z.; Zhang, J.; Jin, R.; Aboagye-Nimo, E. Employees' safety perceptions of site hazard and accident scenes. J. Constr. Eng. Manag. 2018, 145, 04018117. [CrossRef]

27. Han, Y.; Jin, R.; Wood, H.; Yang, T. Investigation of Demographic Factors in Construction Employees' Safety Perceptions. KSCE J. Civ. Eng. 2019, 1-14. [CrossRef]

28. Tang, N.; Hu, H.; Xu, F.; Zhu, F. Personalized safety instruction system for construction site based on internet technology. Saf. Sci. 2019, 116, 161-169. [CrossRef]

29. Razkenari, M.; Qi, B.; Fenner, A.; Hakin, H.; Costin, A.; Kibert, C. Industrialized Construction: Emerging Methods and Technologies. In Proceedings of the International Conference on Computing in Civil Engineering (i3CE), Atlanta, GA, USA, 17-19 June 2019; ASCE: Reston, VA, USA; pp. 352-359.

30. Cheng, C.; O'Sullivan, B. When Artificial Intelligence Meets the Construction Industry. 2017. Available online: https://www.volvoce.com/global/en/news-and-events/company-stories/when-artificial-intelligencemeets-the-construction-industry/ (accessed on 25 May 2018).

31. Tang, S.; Shelden, D.R.; Eastman, C.M.; Pishdad-Bozorgi, P.; Gao, X. A review of building information modeling (BIM) and the internet of things (IoT) devices integration: Present status and future trends. Autom. Constr. 2019, 101, 127-139. [CrossRef]

32. Smartvid.io, Inc. Smartvid.io. 2018. Available online: https://www.smartvid.io (accessed on 27 May 2018).

33. Qi, B.; Ke, C.; Costin, A. RFID and BIM-Enabled Prefabricated Component Management System in Prefabricated Housing Production; Construction Research Congress: New Orleans, LA, USA, 2018.

34. Nichols, G. Robots Are Coming to Work. Are They Safe? ZDNet. 2017. Available online: https: //www.zdnet.com/article/robots-are-coming-to-work-are-they-safe/ (accessed on 22 May 2018).

35. Costin, A.; Pradhananga, N.; Teizer, J. Leveraging Passive RFID Technology for Construction Resource Field Mobility and Status Monitoring in a High-Rise Renovation Project. Autom. Constr. 2012, 24, 1-15. [CrossRef]

36. Costin, A.; Teizer, J.; Schoner, B. RFID and BIM-Enabled Worker Location Tracking to Support Real-time Building Protocol Control and Data Visualization on a Large Hospital Project. J. Inf. Technol. Constr. (ITcon) 2015, 40, 495-517.

37. Thibaud, M.; Chi, H.; Zhou, W.; Piramuthu, S. Internet of Things (IoT) in high-risk Environment, Health and Safety (EHS) industries: A comprehensive review. Decis. Support Syst. 2018, 108, 79-95. [CrossRef]

38. Haddad, H.; Bouyahia, Z.; Chaudhry, S.A. A Multiagent Geosimulation and IoT-based Framework for Safety Monitoring in Complex Dynamic Spatial Environments. Procedia Comput. Sci. 2019, 151, 527-534. [CrossRef] 
39. Kanan, R.; Elhassan, O.; Bensalem, R. An IoT-based autonomous system for workers' safety in construction sites with real-time alarming, monitoring, and positioning strategies. Autom. Constr. 2018, 88, 73-86. [CrossRef]

40. Zhang, M.; Cao, T.; Zhao, X. Applying sensor-based technology to improve construction safety management. Sensors 2017, 17, 1841. [CrossRef]

41. Zhang, M.; Chen, S.; Zhao, X.; Yang, Z. Research on construction workers' activity recognition based on smartphone. Sensors 2018, 18, 2667. [CrossRef]

42. Rossi, A.; Vila, Y.; Lusiani, F.; Barsotti, L.; Sani, L.; Ceccarelli, P.; Lanzetta, M. Embedded smart sensor device in construction site machinery. Comput. Ind. 2019, 108, 12-20. [CrossRef]

43. Park, J.; Marks, E.; Cho, Y.K.; Suryanto, W. Performance test of wireless technologies for personnel and equipment proximity sensing in work zones. J. Constr. Eng. Manag. 2015, 142, 04015049. [CrossRef]

44. Zou, P.X.; Lun, P.; Cipolla, D.; Mohamed, S. Cloud-based safety information and communication system in infrastructure construction. Saf. Sci. 2017, 98, 50-69. [CrossRef]

45. Martinez-Aires, M.D.; Lopez-Alonso, M.; Martinez-Rojas, M. Building information modeling and safety management: A systematic review. Saf. Sci. 2018, 101, 11-18. [CrossRef]

46. Fang, Y.; Cho, Y.K.; Durso, F.; Seo, J. Assessment of operator's situation awareness for smart operation of mobile cranes. Autom. Constr. 2018, 85, 65-75. [CrossRef]

47. Soltanmohammadlou, N.; Sadeghi, S.; Hon, C.K.; Mokhtarpour-Khanghah, F. Real-time locating systems and safety in construction sites: A literature review. Saf. Sci. 2019, 117, 229-242. [CrossRef]

48. Xu, Q.; Chong, H.Y.; Liao, P.C. Collaborative information integration for construction safety monitoring. Autom. Constr. 2019, 102, 120-134. [CrossRef]

49. Zhou, C.; Luo, H.; Fang, W.; Wei, R.; Ding, L. Cyber-physical-system-based safety monitoring for blind hoisting with the internet of things: A case study. Autom. Constr. 2019, 97, 138-150. [CrossRef]

50. Jacobs, J.V.; Hettinger, L.J.; Huang, Y.H.; Jeffries, S.; Lesch, M.F.; Simmons, L.A.; Willetts, J.L. Employee acceptance of wearable technology in the workplace. Appl. Ergon. 2019, 78, 148-156. [CrossRef]

51. Korman, D.B.; Zulps, A. Enhancing Construction Safety Using Wearable Technology; American Society of Safety Engineers: Des Plaines, IL, USA, 2017.

52. Sigcha, L.; Pavón, I.; Arezes, P.; Costa, N.; De Arcas, G.; López, J. Occupational Risk Prevention through Smartwatches: Precision and Uncertainty Effects of the Built-In Accelerometer. Sensors 2018, 18, 3805. [CrossRef]

53. Hopkins, A. Thinking about process safety indicators. Saf. Sci. 2009, 47, 460-465.

54. Reiman, T.; Pietikäinen, E. Leading indicators of system safety-Monitoring and driving the organizational safety potential. Saf. Sci. 2012, 50, 1993-2000. [CrossRef]

55. Costin, A.; McNair, J. IoT-ACRES: Building an IoT Infrastructure for Analysis of Construction and Real Estate Sustainability, Technical Report; University of Florida: Gainesville, FL, USA, 2019; pp. 1-5.

56. Costin, A.; Eastman, C. Need for Interoperability to Enable Seamless Information Exchanges in Smart and Sustainable Urban Systems. J. Comput. Civ. Eng. 2019, 33. [CrossRef]

57. OSHA. Commonly Used Statistics. United States Department of Labor, 2019. Available online: https: //www.osha.gov/oshstats/commonstats.html (accessed on 1 October 2019).

58. OSHA. Heat Stress; United States Department of Labor: Washington, DC, USA, 2019. Available online: https://www.osha.gov/SLTC/heatstress/ (accessed on 1 October 2019).

59. Anderson, M. 6 Key Findings on How Americans See the Rise of Automation; Pew Research Center: Washington, WA, USA, 2017. Available online: http://www.pewresearch.org/fact-tank/2017/10/04/6-key-findings-on-howamericans-see-the-rise-of-automation/ (accessed on 20 May 2018).

60. Cambraia, F.B.; Saurin, T.A.; Formoso, C.T. Identification, analysis and dissemination of information on near misses: A case study in the construction industry. Saf. Sci. 2010, 48, 91-99. [CrossRef]

61. Van Der Schaaf, T.; Kanse, L. Biases in incident reporting databases: An empirical study in the chemical process industry. Saf. Sci. 2004, 42, 57-67. [CrossRef] 
62. Winkler, M.; Perlman, Y.; Westreich, S. Reporting near-miss safety events: Impacts and decision-making analysis. Saf. Sci. 2019, 117, 365-374. [CrossRef]

63. Schukat, M.; McCaldin, D.; Wang, K.; Schreier, G.; Lovell, N.H.; Marschollek, M.; Redmond, S.J. Unintended Consequences of Wearable Sensor Use in Healthcare. Contribution of the IMIA Wearable Sensors in Healthcare WG. Yearb. Med Inform. 2016, 25, 73-86. [CrossRef] 